\title{
Externe Elemente der Corporate Governance von Stiftungen und Vereinen im internationalen Vergleich. Implikationen aus Österreich und der Schweiz für Reformansätze in Deutschland
}

\author{
Nonprofit Governance, Verein, Stiftung, Rechnungslegung, Prüfung, Publizität, Aufsicht
}

Der vorliegende Beitrag soll Reformansätze zur Verbesserung der externen Elemente der Corporate Governance von Stiftungen und Vereinen aufzeigen, da im deutschen Recht nur geringe Anforderungen an deren Rechnungslegung, Prüfung, Publizität und Aufsicht gestellt werden. Hierzu wird eine Analyse der entsprechenden Aspekte für die Länder Österreich und Schweiz vorgenommen, um Implikationen für Reformansätze der beiden Rechtsformen in Deutschland herauszuarbeiten. Im Ergebnis zeigt sich, dass eine angemessene und verlässliche Berichterstattung notwendig ist, um das Vertrauen in Nonprofit-Organisationen zu stärken.

\section{Einleitung}

Nonprofit-Organisationen (NPO), wie bspw. Stiftungen und Vereine, dienen innerhalb einer Volkswirtschaft dazu, eine Lücke zwischen Markt und Staat zu schließen, indem sie Leistungen erbringen, die von den beiden vorgenannten Instanzen nicht oder nur in unzureichender Menge angeboten werden (Walz 2002, S. 269). Im Gegensatz zu wirtschaftlich orientierten Gesellschaften liegt die Zweckausrichtung von NPO nicht in der Erwirtschaftung von Erträgen, sondern in der Erfüllung ihrer individuellen Aufgabe. Dabei verfolgen sie oft gemeinnützige Ziele wie die Bereitstellung von Dienstleistungen sowie die finanzielle Unterstützung und Durchführung sozial wertvoller Projekte. (Schneider/Minnig/Freiburghaus 2007, S. 17-18). Der Nutzen dieses sog. Dritten Sektors liegt für eine Volkswirtschaft dabei nicht nur in seiner bedeutenden sozialen Sachleistung, sondern darüber hinaus in seinem wirtschaftlichen Leistungsbeitrag. NPO tragen in einem nicht unerheblichen Maße zur Wertschöpfung und Erwirtschaftung des Bruttoinlandproduktes (BIP) bei und stellen in Deutschland viele Arbeitsplätze sowohl im ehrenamtlichen als auch im professionellen Bereich zur Verfügung (Anheier et al. 2007, S. 17-39). Die Rechtsformen des Vereins und der Stiftung sind im deutschen NPO-Sektor am häufigsten vertreten (Priller/ Zimmer 2001, S. 15). Der Bestand an (nicht) eingetragenen Vereinen und (nicht) rechtsfähigen Stiftungen in Deutschland wird auf ca. 1 Mio. geschätzt (Strachwitz 2004, S. 206). Da der Sektor kaum in amtlichen Statistiken erfasst wird, ist die genaue Größe schwer zu eruieren.

1 Dipl.-Kffr. Ira Führer ist Mitarbeiterin bei der REpower Systems SE, Hamburg. Dr. Remmer Sassen ist wissenschaftlicher Mitarbeiter am Institut für Wirtschaftsprüfung und Steuerwesen, Lehrstuhl für Revisions- und Treuhandwesen, Universität Hamburg (Prof. Dr. Carl-Christian Freidank). 
Die vorliegende Analyse knüpft an einen Beitrag der Verfasser zum Reformbedarf externer Elemente der Corporate Governance von Stiftungen an (Führer/Sassen 2010, S. 227-236). Hier wird ausführlich gezeigt, „dass das deutsche Recht nur wenige Anforderungen an die Rechnungslegung, Transparenz und Prüfung von Stiftungen legt, die zudem bundesweit sehr uneinheitlich ausgestaltet sind.“ (Führer/Sassen 2010, S. 235). Die Anforderungen an Vereine sind in Deutschland noch geringer ausgeprägt. Ziel ist es, vor dem Hintergrund der internationalen Praxis Reformansätze im deutschen Stiftungs- und Vereinsrecht aufzuzeigen, die der weiteren Entwicklung der externen Corporate Governance-Komponenten dienen und damit den Schutz der öffentlichen Interessen gewährleisten sollen. Hierzu wird in Abschnitt II der Reformbedarf des Stiftungs-und Vereinsrechts in Deutschland dargelegt. Nach der Analyse der stiftungs- und vereinsrechtlichen Gestaltung im österreichischen Recht (Abschnitt III), werden sodann in Abschnitt IV die entsprechenden schweizerischen Regelungen vorgestellt. Der Beitrag schließt mit einem Fazit ab.

\section{Reformbedarf des Stiftungs- und Vereinsrechts in Deutschland}

Wie der Markt und der Staat kann auch der Dritte Sektor versagen. Aufgrund seiner gemeinwohltätigen Zielsetzungen ist er nicht automatisch vor kriminellen Handlungen gefeit. Außerdem mangelt es im Vergleich zum Wirtschaftssektor, dessen Funktion grundsätzlich durch Marktkräfte gewährleistet wird, dem Dritten Sektor an derartigen Sicherungsmechanismen. Wenn das Vertrauen in die Organisationen verloren geht, werden Spender und Mitglieder zwar ihr Engagement einschränken, doch fehlt es an umfassenden Kontrollmöglichkeiten und Transparenz über die Qualität und weitgehend auch die Quantität der Leistung (Schneider/Minnig/Freiburghaus 2007, S. 21-22). Die Notwendigkeit der Implementierung von angemessenen und funktionsfähigen Überwachungsinstrumenten zur Schaffung von Sicherheit für die Öffentlichkeit wurde in der Vergangenheit durch diverse Vertrauensskandale mehr als verdeutlicht. Veruntreuungsaffären mit Spendengeldern in Millionenhöhe gab es bspw. bei der Caritas-Trägergesellschaft-Trier im Jahr 2000 oder beim Deutschen Tierhilfswerk e. V. im Jahr 2003. Der Entzug des Spenden-Siegels des UNICEF e. V. in 2008 durch das Deutsche Zentralinstitut für soziale Fragen (DZI) sorgte ebenfalls für eine negative Öffentlichkeitswirkung.

Die in- und externe Überwachung von NPO kann zur Wahrung der öffentlichen Interessen beitragen. Neben internen Kontrolleinrichtungen und Anreizmechanismen zur zielgerichteten Vereins- und Stiftungsführung sind ebenfalls externe Überwachungskomponenten zu den Elementen der Corporate Governance zu subsumieren (Freidank/Velte 2008, S. 713). Die gesetzlich normierten, externen Überwachungsinstrumente, wie die externe Berichterstattung, Prüfung, Publizität und Rechtsaufsicht von Stiftungen und Vereinen, sowie der Nutzen dieser Vorschriften zur Sicherung und Verbesserung der existentiellen Unterstützung durch die Allgemeinheit stehen im Vordergrund der Analyse.

Durch verbindliche Vorgaben zur Ausgestaltung der Berichterstattung kann der Ermessensspielraum im Rahmen der Rechenschaftsablegung gegenüber externen Adressaten auf ein Minimum reduziert werden. Die weitreichende Transparenz relevanter Informationen mittels Berichterstattung, als wesentliche Voraussetzung zur Beurteilung der Effizienz und Effektivität der Stiftungs-/ Vereinsführung, hilft die Informationsasymmetrien zwischen den Akteuren gezielt zu verringern 
und opportunistische Handlungen weitgehend zu vermeiden (Paetzmann 2007, S. 303). Die Prüfung der Rechnungslegung durch außenstehende Dritte stärkt das öffentliche Vertrauen in das Management (Werder 2003, S. 14). Zudem erhöht die Gewährleistung der Richtigkeit finanzieller Daten den Schutz der Auskünfte im Rahmen von Publizitätspflichten. Durch die Veröffentlichung (Publizität) der geprüften wirtschaftlichen Situation der Organisation wird der Kenntnisstand der externen Personengruppen verbessert und die internen Bezugsgruppen werden daran gehindert, ihr Wissen für eigene Zwecke und zu Lasten der Stakeholder auszunutzen (Schneider/Bienek 2004, S. 35). Weiterhin ist die staatliche Aufsicht zum Corporate Governance-Instrumentarium zu zählen. Sie setzt gesetzliche Vorgaben durch und dient dazu, fehlende Eigenkontrollen zu ersetzen bzw. bestehende Überwachungsmechanismen zu ergänzen, um die Umsetzung einer angemessenen Corporate Governance zu gewährleisten (Hopt 2005, S. 250).

Das deutsche Recht legt nur wenige Anforderungen an die Rechnungslegung, Transparenz und Prüfung von Stiftungen und Vereinen. So ist der Vorstand eines Vereins lediglich dazu verpflichtet in der Mitgliederversammlung Rechenschaft abzulegen und eine Einnahmen-Ausgaben-Rechnung (EAR) zu erstellen. Das Vereinsrecht ist insoweit auch auf Stiftungen anzuwenden. Offenlegungs- und Prüfungspflichten existieren bei beiden Gesellschaftsformen kaum. Allerdings werden die Rechnungslegungs- und Publizitätspflichten für Stiftungen durch Landesstiftungsgesetze ausgedehnt. Sie schreiben vor, dass den Aufsichtsbehörden jährlich eine Jahres(ab)rechnung inkl. eines Vermögens- und Tätigkeitsberichtes vorzulegen ist. Die Aufsichtsämter halten sich außerdem mehr oder weniger umfangreiche Prüfungsmöglichkeiten vor. In Fällen von erwerbswirtschaftlich tätigen Vereinen und Stiftungen hingegen erweitern sich die gesetzlichen Anforderungen an die Rechnungslegung, Transparenz und ggf. auch an die Prüfung. Die Grenzen zur verpflichtenden Rechnungslegung nach den HGB-Standards und eine Kontrolle durch Wirtschaftsprüfer sind im PublG jedoch derart hoch angesetzt, dass sie lediglich auf einen Bruchteil von Organisationen des Nonprofit-Sektors Anwendung finden. Das PublG gilt gem. §3 Abs. 1. Nr. 3, Nr. 4 PublG i. V. m. § 1 PublG für Stiftungen und Vereine, wenn sie an zwei aufeinander folgenden Abschlussstichtagen jeweils mindestens zwei der drei nachstehenden Merkmale erfüllen:

- Bilanzsumme übersteigt 65 Mio. Euro.

- Umsatzerlöse übersteigen 130 Mio. Euro.

- Beschäftigung von durchschnittlich mehr als 5.000 Arbeitnehmern. 
Abbildung 1 zeigt zusammenfassend die bundesgesetzlichen Vorschriften auf.

\begin{tabular}{|c|c|c|c|c|c|}
\hline & \multicolumn{3}{|c|}{ Rechnungslegungspflicht } & \multirow{3}{*}{ Prüfungspflicht } & \multirow{3}{*}{$\begin{array}{l}\text { Publizitätspflicht der } \\
\text { Rechnungslegung }\end{array}$} \\
\hline & \multirow[b]{2}{*}{ EAR } & \multicolumn{2}{|c|}{ kaufmännisch eingerichtetes Rechnungswesen } & & \\
\hline & & $\begin{array}{c}\text { handelsrechtliche Vorschriften } \\
\text { für Kaufleute }\end{array}$ & $\begin{array}{l}\text { handelsrechtliche Vorschriften für } \\
\text { Kapitalgesellschaften }\end{array}$ & & \\
\hline 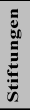 & $\begin{array}{c}\text { Pflicht } \\
(\S 86 \text { BGB i.V.m. } \\
\S \S 27 \text { Abs. } 3 \text { und } 666 \text { BGB })\end{array}$ & $\begin{array}{l}\text { Handelsgewerbe } \\
\text { i.S.d. } \$ \S 1-3 \mathrm{HGB}\end{array}$ & $\begin{array}{l}\text { Stiftungen mit Gewerbebetrieb } \\
\qquad(\$ 5 \text { PublG i.V.m } \\
\S \S 1 \text { und } 3 \text { Abs. } 1 \text { Nr. } 4 \text { PublG) }\end{array}$ & $\begin{array}{l}\text { Stiftungen mit Gewerbebetrieb } \\
\qquad(\$ 6 \text { PublG i.V.m } \\
\S \S 1 \text { und } 3 \text { Abs. } 1 \text { Nr. } 4 \text { PublG) }\end{array}$ & $\begin{array}{l}\text { Stiftungen mit Gewerbebetrieb } \\
(\$ 9 \text { PublG i.V.m. } \\
\$ \S 1 \text { und } 3 \text { Abs. } 1 \text { Nr. } 4 \text { PublG) }\end{array}$ \\
\hline 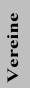 & $\begin{array}{c}\text { Pflicht } \\
(\S 27 \text { Abs. } 3 \text { BGB i.V.m. } \\
\S 666 \text { BGB })\end{array}$ & $\begin{array}{l}\text { Handelsgewerbe } \\
\text { i.S.d. } \$ \S 1-3 \mathrm{HGB}\end{array}$ & $\begin{array}{c}\text { Wirtschaftsvereine } \\
(\$ 5 \text { PublG i.V.m } \\
\S \S 1 \text { und } 3 \text { Abs. } 1 \text { Nr. } 3 \text { PublG })\end{array}$ & $\begin{array}{c}\text { Wirtschaftsvereine } \\
(\$ 6 \text { PublG i.V.m } \\
\S \S 1 \text { und } 3 \text { Abs. } 1 \text { Nr. } 3 \text { PublG) }\end{array}$ & $\begin{array}{c}\text { Wirtschaftsvereine } \\
(\$ 9 \text { PublG i.V.m } \\
\S \S 1 \text { und } 3 \text { Abs. } 1 \text { Nr. } 3 \text { PublG) }\end{array}$ \\
\hline
\end{tabular}

Abbildung 1: Überblick über die stiftungs- und vereinsrechtlichen bundesgesetzlichen Vorschriften zur externen Corporate Governance

Quelle: Eigene Darstellung

In Deutschland werden verbindliche und weitergehende Regelungen zur Einführung einer angemessenen Nonprofit-Rechnungslegung, Publizität und Prüfung benötigt, um eine Vergleichbarkeit innerhalb des deutschen Nonprofit-Sektors herstellen und die Informationsinteressen der Anspruchsgruppen befriedigen zu können. Die Vorschriften zur Rechnungslegung und Transparenz sowie die Prüfung der relevanten Daten von Stiftungen (Führer/Sassen 2010, S. 227-236) und Vereinen befinden sich in Deutschland auf einem sehr niedrigen Niveau. Dies wird besonders im Vergleich zur internationalen Praxis deutlich. Die österreichischen und schweizerischen Nonprofit-Forschungen haben in den 1980er Jahren das deutschsprachige Gebiet stark geprägt (Löwe 2003, S. 1). Beide Länder haben zudem jüngst ihr Stiftungs- und Vereinsrecht, speziell im Hinblick auf die finanzielle Kontrolle, umfassend reformiert und an die aktuellen (wirtschaftlichen) Entwicklungen im Dritten Sektor angepasst. Dies ist in Deutschland bislang nicht geschehen. Um Verbesserungsmöglichkeiten aufzuzeigen, damit erfolgversprechende Alternativen ggf. in das deutsche Recht übernommen werden können, wird nachfolgend eine Darstellung der stiftungsund vereinsspezifisschen Regelungen der Länder Österreich und Schweiz vorgenommen.

\section{Stiftungs- und Vereinsrecht in Österreich}

\section{Rahmenbedingungen}

\section{a) Stiftungsrecht}

Das österreichische Stiftungswesen lässt sich in öffentlich-rechtliche und private Stiftungen einteilen. Der Zweck einer öffentlich-rechtlichen Stiftung muss, im Gegensatz zum Ziel einer Privatstiftung, gem. $\S 1$ Abs. 1 und $\S 5$ Abs. 1 Nr. 2 Bundes-Stiftungs- und Fondsgesetz in der Förderung des Gemeinwohls liegen. Eine private Stiftung kann dagegen sowohl eigen- als auch fremdnützige Ziele verfolgen (Lang/Rief 1994, S. 147). Das Bundes-Stiftungs- und Fondgesetz gilt ausschließlich für öffentlich-rechtliche Stiftungen. Vergleichbar mit dem deutschen Recht 
entstehen solche wohltätigen Stiftungen gem. § 3 Bundes-Stiftungs- und Fondsgesetz durch Zustimmung der zuständigen Behörden und unterliegen deren Kontrolle. Da aus dem Bundes-Stiftungs- und Fondgesetz heraus keine eigennützlichen Stiftungen gegründet werden dürfen, trat 1993 das Privatstiftungsgesetz (PSG) in Kraft, um insbesondere drohende Vermögensabwanderungen ins Ausland und den damit einhergehenden Verlust von Steuergeldern zu vermeiden (Simsa 2006, S. 143). Die folgenden Analysen konzentrieren sich auf die österreichische Privatstiftung.

Gem. $§ 1$ Abs. 1 PSG ist unter einer Stiftung ,[...] ein Rechtsträger [zu verstehen], dem vom Stifter ein Vermögen gewidmet ist, um durch dessen Nutzung, Verwaltung und Verwertung der Erfüllung eines erlaubten, vom Stifter bestimmten Zwecks zu dienen [...]“. Die Errichtung einer Stiftung erfolgt im Rahmen einer notariell beglaubigten Stiftungserklärung, die gem. $§ 9$ Abs. 1 PSG unter anderem den Stiftungsnamen, und -sitz, den Stiftungszweck und eine Beschreibung der zu begünstigenden Personen enthält. Die Rechtspersönlichkeit erhält die Stiftung mittels Eintragung in das Firmenbuch und Aufbringung des erforderlichen Mindestkapitals in Höhe von 70.000 Euro gem. §§ 4, 7, 12 PSG (Simsa 2006, S. 144; Nowotny/Fida 2007, S. 227). Beim Firmenbuch handelt es sich um das österreichische Handelsregister (Doralt 1996, S. 5).

Neben dem Vorstand, der zur Stiftungsleitung und Erfüllung des Stiftungszweckes nach $\S 17$ Abs. 1 PSG verpflichtet ist, zählt gem. § 14 Abs. 1 PSG der Stiftungsprüfer zu den Stiftungsorganen sowie ggf. ein Aufsichtsrat, der vom Gericht zu bestellen ist und die Stiftungsleitung nach $\S \S 24,25$ PSG zu kontrollieren hat. Allerdings ist dieses Kontrollorgan gem. $§ 22$ Abs. 1 Nr. 1, Nr. 2 PSG nur zu bilden, wenn die Stiftungserklärung dies bestimmt bzw. die durchschnittliche Arbeitnehmeranzahl der Stiftung die Grenze von 300 überschreitet. Darüber hinaus können gem. § 17 Abs. 2 PSG weitere Organe, wie etwa Beiräte oder auch Begünstigten- und Stifterversammlungen, die in der Stiftungserklärung vom Stifter zu benennen sind, gebildet werden, um die Verfolgung des Stiftungszweckes sicherzustellen (o. V. 2007/2008, S. 53).

\section{b) Vereinsrecht}

Das Recht zur Vereinigung und Bildung von Vereinen wird in Österreich durch den Art. 12 Staatsgrundgesetz (StGG) verfassungsrechtlich geschützt. Des Weiteren stellt das im Jahr 2002 umfassend reformierte Vereinsgesetz (VerG) die Rechtsgrundlage zur Bildung, zum Fortbestand und zur Beendigung von Vereinen dar. Das VerG wird durch die Vereinsrichtlinien (VereinsRL) flankiert, die neben der Bundesabgabenordnung (BAO) ebenfalls Grundsätze zur Vereinsbesteuerung enthalten. Darüber hinaus beinhalten unter anderem die Gewerbeordnung sowie das Unternehmensgesetzbuch (UGB) aufgrund der möglichen wirtschaftlichen Betätigung von Vereinen ergänzende Vorschriften (Brändle/Schnetzer 2002, S. 37-43).

Eine Erklärung des Begriffs Verein findet sich in $§ 1$ Abs. 1 VerG. Demnach wird ein Verein als ,[...] freiwilliger, auf Dauer angelegter, aufgrund von Statuten organisierter Zusammenschluss mindestens zweier Personen zur Verfolgung eines bestimmten, gemeinsamen, ideellen Zwecks durch fortgesetzte gemeinschaftliche Tätigkeit[...]“ definiert. Die Gründung erfolgt gem. § 2 Abs. 1 VerG durch einen verfahrensrechtlichen Rechtsakt und vollzieht sich gem. §§ 11-14 VerG in den zwei Schritten der Errichtung (Statutenvereinbarung) und Entstehung (Brändle/Schnetzer 
2002, S. 111-119). Der Inhalt der Vereinsverfassung wird vom österreichischen Gesetzgeber weitgehender vorgeschrieben als dies in Deutschland der Fall ist. Abbildung 2 zeigt hierzu vergleichend die gesetzlichen Vorschriften.

\begin{tabular}{|c|c|}
\hline $\begin{array}{c}\text { Mindestinhalt der Vereinssatzung } \\
\$ 3 \text { Abs. } 2 \text { VerG }\end{array}$ & Deutsches Recht \\
\hline $\begin{array}{l}\text { - Name } \\
\text { - Sitz } \\
\text { - klare \& umfassende Zweckbeschreibung }\end{array}$ & $\begin{array}{c}\text { Pflicht } \\
\text { (§57 Abs. 1 BGB) }\end{array}$ \\
\hline $\begin{array}{l}\text { - Erwerb \& Beendigung der Mitgliedschaft } \\
\text { - } \text { Mitgliederrechte \& -pflichten } \\
\text { - } \text { tretung \& deren Aufgaben, insbesondere Geschäftsführung \& Ver- } \\
\text { - } \\
\text { - Ert der Organbestellung \& Amtsdauer } \\
\text { Erfornisse für Beschlussfassung }\end{array}$ & $\begin{array}{l}\text { Soll-Bestimmung } \\
\quad(\S 58 \mathrm{BGB})\end{array}$ \\
\hline $\begin{array}{l}\text { - Schlichtungsart von Streitigkeiten } \\
\text { - Bestimmungen über eine freiwillige Auflösung \& } \\
\text { - Vermögensverwendung } \\
\text { - vorgesehene Tätigkeit sowie Art \& Aufbringung der benötigten Mit- } \\
\text { tel }\end{array}$ & keine Vorgaben \\
\hline
\end{tabular}

\section{Abbildung 2: Inhalt der österreichischen Vereinsstatuten im Vergleich zum deutschen Recht}

Quelle: Eigene Darstellung

Die Rechtspersönlichkeit wird im Rahmen einer Gründungsanzeige bei der Vereinsbehörde erlangt, wenn die Bildung nach Ablauf einer vierwöchigen Frist gem. § 13 Abs. 1 VerG nicht untersagt wird, (Nowotny/Fida 2007, S. 215-216). Die Organe eines Vereins bestehen laut $\S 5$ Abs. 1 VerG aus der Mitgliederversammlung, dem obersten Organ zur Willensbildung und der Geschäftsführung, die den Verein leitet und vertritt. Darüber hinaus muss eine Einrichtung zur Schlichtung von Konflikten, eine sog. Schlichtungsstelle, in den Statuten vorgesehen werden, die gem. $\S 8$ VerG ebenfalls zu den Vereinsorganen zählt. Zusätzlich können die Statuten die Bildung eines Aufsichtsorgans bestimmen, das durch die Mitgliederversammlung bestellt wird und in ihrem Auftrag die Tätigkeit des Leitungsorgans kontrolliert. Bei entsprechend großen Vereinen mit durchschnittlich mehr als 300 Arbeitnehmern ist das Aufsichtsorgan gem. § 5 Abs. 4 VerG i. V.m. $\S \S 132,110$ ArbVG ebenfalls paritätisch zu besetzen, es sei denn, der Verein verfolgt karitative, wissenschaftliche, erzieherische oder ähnliche Zwecke.

Der Hinweis auf eine ideelle Zweckverfolgung in der gesetzlichen Definition stellt klar, dass es sich nicht um einen Wirtschaftsverein handeln darf. Die das Gesetz umfassenden Vereine sind demnach nicht gewinnorientiert ausgerichtet, dürfen allerdings in einem gewissen Umfang wirtschaftlich tätig sein. Da hierzu keine gesetzlichen Regelungen getroffen wurden, gibt es in der österreichischen Rechtsprechung verschiedene Standpunkte, wie weit eine wirtschaftliche Betätigung zu fassen ist. Der Verfassungsgerichtshof (VfGH) und die Erläuterungen zum VerG tolerieren eine wirtschaftliche Tätigkeit, soweit überschüssige Gewinne nicht zweckwidrig genutzt oder an Dritte verteilt werden (Hippel 2007, S. 514-516). Somit geht die Erlaubnis hier sehr viel weiter als es im deutschen Recht der Fall ist, da eine unbegrenzte wirtschaftliche Betätigung 
möglich wird. Die Auffassung des Obersten Gerichtshofes von Österreich (OGH) hingegen ist mit der deutschen Anerkennung der wirtschaftlichen Tätigkeit vergleichbar, da dessen Urteile auf das Nebenzweckprivileg abstellen. Nach diesem zielgerichteten Ansatz muss die wirtschaftliche Betätigung dem ideellen Zweck untergeordnet sein und darf dem Verein bzw. dessen Mitgliedern nicht als Selbstzweck dienen (Brändle/Schnetzer 2002, S. 67-79). Das auch durch den wirtschaftlichen Bereich erzielte Vermögen darf nur für den festgelegten Vereinszweck genutzt werden. Die möglichen Tätigkeiten zur Zweckverwirklichung und Vermögensverwendung sind gem. § 1 Abs. 2 VerG einzeln in den Statuten auszuweisen.

Im Rahmen der Vereinsrechtsreform wurden neben erhöhten Rechnungslegungs- und Prüfungspflichten auch die Offenlegungsvorschriften weitgehend umgestaltet und verbessert. Wie es auch in anderen europäischen Staaten, z. B. Frankreich oder Großbritannien üblich ist, hat der österreichische Gesetzgeber zur Transparenzförderung und verstärkten Kontrollmöglichkeit erstmals eine Verpflichtung zur kaufmännischen Rechnungslegung und Prüfung verbindlich festgelegt (Donschen 2008, S. 99; Walz 2005, S. 275). Vor dem Hintergrund der verstärkten wirtschaftlichen Betätigung von Vereinen wurden die Anforderungen an die Ausgestaltung des Rechnungswesens und die Prüfungspflichten gem. $\S \S 21,22$ VerG an die Größe der Vereine gebunden, um die rechtsformspezifischen Besonderheiten angemessen zu berücksichtigen (Ebers 2005, S. 71-72). Diese Regelungen werden zusammen mit den entsprechenden Regelungen für Stiftungen im Folgenden ausführlich dargestellt.

\section{Externe Corporate Governance-Instrumente von Stiftungen und Vereinen}

\section{a) Rechnungslegung}

Die Pflichten zur Rechnungslegung sind im österreichischen Stiftungsrecht klar und übersichtlich ausgestaltet. Entsprechend wird jede Privatstiftung einem Vollkaufmann gleichgestellt und ist so zu einer umfassenden Rechnungslegung verpflichtet. Gem. § 18 PSG i. V. m. §§ 189-216, 222-234, 236-239, 243-267 UGB muss neben einem kaufmännischen Jahresabschluss ein Lagebericht erstellt werden, der insbesondere die Zweckerfüllung zum Gegenstand hat. Die für Kapitalgesellschaften geltenden Vorschriften zur Aufstellung von Bilanz, Gewinn- und Verlustrechnung $(\mathrm{GuV})$, Anhang und Lagebericht gelten größenunabhängig für alle Stiftungen.

Die externe Rechnungslegung der österreichischen Vereine ist in Abhängigkeit zur Größe ausgestaltet. Abbildung 3 zeigt die Größeneinordnung, wobei die Überschreitung der jeweiligen Grenzen gem. $\S 22$ Abs. 1 und 2 VerG an zwei aufeinander folgenden Stichtagen erfolgen muss. 
Externe Elemente der Corporate Governance von Stiftungen und Vereinen im internationalen Vergleich

\begin{tabular}{|l|c|c|}
\hline & Einnahmen/Ausgaben & Spendeneinnahmen \\
\hline Kleine Vereine & $\leq 1$ Mio. Euro & - \\
\hline Mittelgroße Vereine & $>1$ Mio. Euro & - \\
\hline Große Vereine & $\geq 3$ Mio. Euro & $>1$ Mio. Euro \\
\hline
\end{tabular}

\section{Abbildung 3: Größenkriterien österreichischer Vereine}

Quelle: Eigene Darstellung

Als Mindestanforderung hat der Vorstand kleiner Vereine gem. § 21 Abs. 2 VerG eine Einnahmen-Ausgaben-Rechnung (EAR) inkl. einer Vermögensübersicht für jedes Geschäftsjahr innerhalb von fünf Monaten nach Jahresende zu erstellen. Die Vermögensübersicht wird in einen monetär bewerteten Abschnitt, der das Guthaben sowie die Verbindlichkeiten und Forderungen beinhaltet und einen unbewerteten Teil gegliedert, der lediglich eine Auflistung der Vermögensgegenstände ohne Bewertung enthält. Das Sachanlagevermögen wird somit grundsätzlich nur mengenmäßig erfasst, wobei eine wertmäßige Erfassung nicht ausgeschlossen ist (Grünberger 2004, S. 120). Die Rechnungslegung wird von der Mitgliederversammlung entgegengenommen und auch von dieser genehmigt. Insoweit kommt der Versammlung ein höheres Gewicht zu als es in deutschen Vereinen der Fall ist (Brändle/Schnetzer 2002, S. 133). Diese Anforderungen an die Ausgestaltung der Vereinsrechnungslegung erhöhen sich mit entsprechender Vereinsgröße, wobei jedoch nur auf die gewöhnlichen Einnahmen oder Ausgaben und nicht auf das gesamte Vereinsvermögen abzustellen ist (Grünberger 2004, S. 122). § 22 VerG normiert entsprechend weitergehende Regelungen für eine qualifizierte Rechnungslegung für Vereine mit beträchtlicher finanzieller Kraft. Demnach sind mittelgroße Vereine gem. § 22 Abs. 2 VerG zu einer Aufstellung eines handelsrechtlichen Jahresabschlusses i. S. d. §§ 189-216 UGB verpflichtet. Großvereine werden den Kapitalgesellschaften gleichgestellt. Entsprechend müssen sie gem. § 22 Abs. 3 VerG i. V. m. $\S$ 189-243 UGB einen erweiterten Jahresabschluss inkl. Anhang, der insbesondere die Einnahmequellen und die entsprechenden Aufwendungen enthält, erstellen. Die Aufstellung eines Lageberichtes hingegen ist nicht erforderlich (Nowotny/Fida 2007, S. 217).

\section{b) Prüfung}

Die kaufmännische Rechnungslegung der Privatstiftungen untersteht in Österreich gem. $§ 20$ Abs. 2 PSG der Überwachung und Kontrolle eines Stiftungsprüfers, wozu ausschließlich beeidete Wirtschaftsprüfer, Steuerberater oder Buchprüfer bzw. deren Gesellschaften berufen werden dürfen. Der Stiftungsprüfer ist laut § 20 Abs. 1 PSG von den zuständigen Gerichten bzw. von einem gebildeten Aufsichtsrat zu bestellen. Die Stiftungsprüfung ist mit der regulären Jahresabschlussprüfung von Wirtschaftsunternehmen zu vergleichen. So normiert § 21 PSG als Prüfungsgegenstand den Jahresabschluss inkl. der Buchhaltung sowie den Lagebericht und verweist bezüglich des Prüfungsumfanges und der Berichterstattung auf die entsprechenden handelsrechtlichen Vorschriften der $\S \S 269$ Abs. 1, 272-275 UGB. Die Prüfberichte werden gem. § 21 Abs. 3 PSG den weiteren Stiftungsorganen zur Einsicht vorgelegt. Neben dieser regulären Jahresabschlussprüfung kann eine Sonderprüfung gerichtlich auf Antrag jedes Stiftungsorgans respektive eines Organ- 
mitglieds angeordnet werden, wenn Verletzungen der Gesetzesbestimmungen oder der Stiftungserklärung vorliegen. In diesem Rahmen werden gem. §31 Abs. 1 und Abs. 2 PSG Ordnungsmäßigkeitsprüfungen zur Wahrung des Stiftungszweckes durchgeführt.

Eine Besonderheit im österreichischen Vereinsrecht ist die nach $§ 5$ Abs. 5 VerG verpflichtende Bestellung von mindestens zwei Rechnungsprüfern, die auch als Organe eingesetzt werden können. Die Rechnungsprüfer können, müssen jedoch nicht notwendigerweise Mitglieder sein. Es ist allerdings sicherzustellen, dass die prüfungsdurchführenden Personen keine Zugehörigkeit oder Abhängigkeit in persönlicher und/oder wirtschaftlicher Hinsicht zu den zu kontrollierenden Organen aufweisen (Brändle/Schnetzer 2002, S. 100-101). Zudem sollte die Mitgliederversammlung im Rahmen der Bestellung darauf achten, dass Mindestqualifikationen wie bspw. buchhalterisches Wissen bestehen (Grünberger 2004, S. 122). Diese internen Revisoren prüfen gem. $\S 21$ Abs. 2 VerG innerhalb von vier Monaten nach Erstellung die Rechnungslegung kleiner und mittelgroßer Vereine auf Ordnungsmäßigkeit sowie satzungsmäßiger Mittelverwendung und bestätigen dies in einem abschließenden Prüfbericht, der der Vereinsleitung sowie einem bestehenden Aufsichtsorgan vorzulegen ist (Brändle/Schnetzer 2002, S. 133-134). Etwaige im Abschlussbericht aufgezeigte Mängel oder bestandsgefährdende Tatsachen müssen gem. §21 Abs. 3, Abs. 4 VerG vom Vorstand beseitigt werden.

Große Vereine i. S. d. $§ 22$ Abs. 2 VerG müssen ihre Rechenschaftslegung durch einen Abschlussprüfer gem. $\S 22$ Abs. 4, Abs. 5 VerG prüfen lassen, der analog zu den Rechnungsprüfern von der Mitgliederversammlung zu bestellen ist (Nowotny/Fida 2007, S. 217-218). Als Abschlussprüfer sind neben Wirtschaftsprüfern ebenfalls Steuerberater und beeidete Buchprüfer zugelassen. Wird im Rahmen der Prüfung festgestellt, dass der Verein gegenwärtig oder auch zukünftig seinen, insbesondere finanziellen, Verpflichtungen nicht nachkommen kann, ist eine Anzeige an die zuständigen Vereinsbehörden vorzunehmen. In diesem Fall wird ein Hinweis im Register aufgenommen, wodurch die Meldung publik gemacht wird. Erst wenn diese Gefährdung ausgeschlossen werden kann, ist der Eintrag durch die zuständige Behörde zu löschen (Grünberger 2004, S. 124).

\section{c) Publizität}

Jeder Destinatär einer Stiftung verfügt über einen umfangreichen Informationsanspruch, der neben der Auskunftserteilung über die Zweckerfüllung auch die Einsicht in das stiftungsrechtliche Rechnungswesen, welches nach $\S 30$ Abs. 1 PSG die Buchführung, den Jahresabschluss und den Lagebericht umfasst, den Prüfungsbericht sowie die Stiftungsurkunde gestattet. Dieses Recht kann gem. § 30 PSG bei Weigerung der Stiftung gerichtlich angeordnet werden. Darüber hinausgehende finanzielle Berichterstattungspflichten gibt es nicht. Ein eigenes Register für Stiftungen ist in Österreich nicht notwendig, da die Stiftungen rechtlich wie Handelsunternehmen eingeordnet werden und gem. $§ 2$ Nr. 11 Firmenbuchgesetz (FBG) i. V. m. § 18 PSG i. V. m. $\S 8,189$ UGB zur Eintragung ins Firmenbuch verpflichtet sind. Die Stiftungsurkunde, welche die Stiftungserklärung enthält, ist somit gem. $\S \S 34,35$ FBG i. V. m. § 9 UGB im Firmenbuch für jedermann einsehbar (Lang/Rief 1994, S. 148). Die fakultativen Angaben der Stiftungserklärung gem. 
$\S 9$ Abs. 2 PSG können jedoch zum Großteil in einer sog. Zusatzurkunde gem. § 10 PSG aufgenommen werden, die keiner Offenlegungspflicht unterliegt.

Im Rahmen der Gesetzesreform im Vereinsrecht ist eine Registerpublizität eingeführt worden, um für weitgehende Transparenz zu sorgen. Es existieren zwei Vereinsregister, auf lokaler und Bundesebene, die über identische Eintragungen relevanter Vereinsdaten verfügen. Die gem. § 16 Abs. 1 VerG vorgegebenen Inhalte unterstehen einer abgestuften Auskunftspflicht. So sind bspw. Name und Nummer des Vereins oder auch Name und Funktion des Vertreters von jedem einsehbar laut $\S 17$ Abs. 1 VerG. Die Anschrift des Vertreters ist gem. § 17 Abs. 2 VerG hingegen nur bei berechtigtem Interesse einsehbar.

Durch den Einsatz dieser Register können alle rechtsrelevanten Daten veröffentlicht werden. Im Gegensatz zum deutschen Recht sind zum einen die Vereine zu mehr Angaben verpflichtet und zum anderen haben diese Eintragungen deklaratorische und keine konstitutive Wirkung, da die Rechtsfähigkeit eines österreichischen Vereins nicht von diesen Eintragungen abhängt (Brändle/ Schnetzer 2002, S. 120). Da es sich um öffentliche Register handelt, steht die Auskunftsberechtigung jeder Person zu, solange keine Auskunftssperre besteht. Diese gem. §17 Abs. 1, Abs. 4 VerG für maximal zwei Jahre bestehende Sperre kann auf Antrag des Vereins eingerichtet werden, wenn die Gefahr besteht, dass sensible Vereinsdaten gefährdet sind und ein Schutzbedürfnis des Vereins begründet ist. Im Rahmen einer rechtlichen Befugnis zur Einsichtnahme sind die Auskünfte gem. $§ 17$ Abs. 6 VerG trotzdessen zu erteilen. Die Registereintragungen verfügen nach $\S 17$ Abs. 8 VerG über einen Vertrauensschutz, so dass die Angaben der Vermutung der Richtigkeit unterliegen, es sei denn die auskunftseinholende Person kennt bzw. muss die Fehlerhaftigkeit der Eintragung kennen (Brändle/Schnetzer 2002, S. 124-128). Auf Bundesebene wird unter der Leitung des Bundesministeriums für Inneres das Zentrale Vereinsregister (ZVR) geführt, wodurch der österreichische Rechtsverkehr erheblich erleichtert und beschleunigt wird, da jede Vereinsbehörde bundesweit auf sämtliche österreichischen Vereinsinformationen zugreifen und diese entsprechend weitergeben kann (Brändle/Schnetzer 2002, S. 128-131).

Neben diesen spezifischen Vereinsregistern sind Vereine gem. $\S 8$ Abs. 1 UGB und $\S 2$ Nr. 11 FBG verpflichtet, Eintragungen im Firmenbuch vorzunehmen, wenn sie Rechnung nach den Vorschriften des $\S 189$ UGB legen müssen. Die einzutragenden Daten entsprechen nach $§ 3$ FBG weitestgehend den Pflichteintragungen des deutschen Handelsregisters. Gem. § 9 Abs. 1 UGB ist jede Person zur Einsicht in das Hauptbuch sowie sonstiger Dokumente berechtigt ( $\S 34,35$ FBG). Die Pflicht zur Auskunftserteilung durch das Leitungsorgan über relevante Vereinsinformationen ist bereits im Jahr 1951 nach Mitgliederprotesten über die mangelnde Informationserteilung im Vereinsgesetz verankert worden. Entsprechend hat der Vorstand gegenüber der im Vier-JahresTurnus stattfindenden Mitgliederversammlung gem. § 20 VerG eine Informationspflicht zu erfüllen, in dessen Rahmen er über die Tätigkeit und die finanzielle Situation des Vereins Auskünfte zu erteilen hat. (Grünberger 2004, S. 125). Durch diesen Rhythmus entsteht zwar durchaus eine periodische Auskunftspflicht, die allerdings mit vier Jahren als sehr lang anzusehen ist. Entsprechend wird im Rahmen des $\S 20$ Satz 2 VerG sichergestellt, dass die Informationsbedürfnisse der Mitglieder auch zwischen den Versammlungen befriedigt werden, indem ihnen ein aperiodisches Informationsrecht auf Anfrage und Interessensbegründung zur Verfügung steht (Brändle/Schnetzer 2002, S. 131-132). Darüber hinaus hat die Vereinsleitung den Mitgliedern Auskunft über das Prüfergebnis zu erteilen. Erfolgt dies in einer Vereinsversammlung, ist gem. § 21 Abs. 4 VerG 
ebenfalls der Rechnungsprüfer einzubeziehen. Mit der Pflicht des Rechnungsprüfers gem. § 21 Abs. 5 VerG eine Mitgliederversammlung einzuberufen, wenn der Vereinsvorstand seiner Rechnungslegungspflicht nicht nachkommt und eine baldige Mängelbeseitigung nicht zu erkennen ist, hat der Gesetzgeber ein zusätzliches Kontrollinstrument geschaffen (Grünberger 2004, S. 121). Neben dieser internen Rechenschaftslegung bestehen keine Verpflichtungen des Vereinsvorstandes zur externen Offenlegung der Rechnungslegung.

\section{d) Aufsicht}

Die staatliche Rechtsaufsicht über Stiftungen üben die zuständigen Gerichte aus, indem sie die Einhaltung der Satzungen und Gesetze überwachen (Nowotny/Fida 2007, S. 228; BMJ 2001, Anlage 3, S. 21). So ist das Gericht gem. § 27 PSG unter anderem für die innere Stiftungsordnung zuständig, indem es fehlende Organe bestellt oder bei Pflichtverletzungen, Unfähigkeit oder im Falle einer Insolvenz bestehende Organe bzw. einzelne Mitglieder abberuft und neu bestellt. Ferner können die österreichischen Gerichte gem. § 35 Abs. 1 Nr. 5 PSG die Stiftungsauflösung beschließen bzw. sind gem. § 35 Abs. 3 PSG dazu verpflichtet, wenn die Zielverfolgung der Stiftung gegen $\S 1$ Abs. 2 PSG verstößt. Eine weitere Kontrolle durch staatliche Aufsichtsbehörden besteht nicht.

Die Aufsicht über österreichische Vereine hinsichtlich der Rechtsaufsicht sowie der Zuständigkeit für Gründung und Auflösung steht der Bezirksverwaltungsbehörde, der allgemeinen österreichischen Verwaltungsstelle des Vereinssitzes und der Bundespolizeidirektion zu. Bei Streitigkeiten und Berufungen gegen die Entscheidungen der ersten Behörden entscheidet gem. § 9 VerG final die Sicherheitsdirektion (Brändle/Schnetzer 2002, S. 107-108). Die Vereinsbehörden prüfen bei Gründung die Vereinssatzungen und entscheiden ggf. über eine Untersagung der Vereinserrichtung, wenn der Zweck, der Name und/oder die innere Verfassung des Vereins gegen Gesetze verstoßen und diese Gesetzeswidrigkeiten zu einer Einschränkung der Vereinsfreiheit führen würden. Ein Vereinsverbot kann allerdings nicht allein aufgrund der Möglichkeit einer zukünftigen rechtswidrigen Betätigung ausgesprochen werden, sondern bedarf gem. §12 Abs. 1 VerG gesetzwidriger Statutenvereinbarungen (Brändle/Schnetzer 2002, S. 112-115)

\section{Stiftungs- und Vereinsrecht in der Schweiz}

\section{Rahmenbedingungen}

\section{a) Stiftungsrecht}

Das in den Art. 80-89 des schweizerischen Zivilgesetzbuches (ZGB) geregelte Stiftungsrecht, wurde 2004 erstmalig seit 1911 reformiert (Purtschert/Schnurbein/Beccarelli 2006, S. 115). Die Neuerungen, die seit dem 1.1.2006 in Kraft sind, betreffen insbesondere die Rechnungslegung und Revision, die Änderungsmöglichkeit der Zwecksetzung und Vermögensübertragungen sowie die Gläubigerschutzvorschriften bei Überschuldung. Im Rahmen einer Überarbeitung des Ge- 
sellschaftsrechts, dessen Novellierungen mit Wirkung vom 1.1.2008 in Kraft getreten sind, haben sich die Vorschriften bezüglich der Stiftungsrechnungslegung und -prüfung sowie der Handelsregistereintragungen erneut geändert, da die Stiftung als Rechtsform in das Obligationenrecht (OR), welches das schweizerische Pendant zum HGB darstellt, aufgenommen worden ist (Jakob 2008, S. 28).

Ebenso wie im deutschen Recht wird die Stiftung im schweizerischen Gesetz nicht näher definiert (Lichtsteiner/Degen/Bärlocher 2008, S. 3). Ihr Zweck ist allerdings nicht auf gemeinnützige Tätigkeiten beschränkt, sondern kann neben privatnützigen Zielsetzungen für sämtliche Aufgaben gegründet werden, solange kein gegen die Sitten oder Gesetze verstoßender Zweck verfolgt wird und eine wirtschaftliche Tätigkeit nicht das Hauptziel der Stiftung darstellt (BMJ 2001, Anlage 3, S. 11). Die Stiftungen werden gem. Art. 81 Abs. 1 ZGB durch eine öffentliche, notariell beglaubigte Urkunde, in der das Stiftungsgeschäft festgehalten wird, und durch Vorlage des Stiftungsentwurfes bei der Eidgenössischen Stiftungsaufsicht, dem Handelsregisteramt und den Steuerbehörden errichtet. Die Errichtung kann auch von Todes wegen aufgrund einer Testamentsverfügung gem. Art. 81 Abs. 1 ZGB erfolgen. Nach einer Prüfung der Stiftungsurkunde durch die vorgenannten Behörden und bei Erfüllung der Mindestvoraussetzungen wird die Stiftung zur Erlangung der Rechtsfähigkeit gem. Art. 81 Abs. 2 ZGB in das Handelsregister eingetragen (Lichtsteiner/Degen/Bärlocher 2008, S. 5). Diese Vorschriften gelten allerdings nicht für kirchliche oder Familienstiftungen (Pavel 1971, S. 67). Somit folgt das schweizerische Stiftungsrecht analog dem österreichischen Recht und entgegen den deutschen Vorschriften dem Normativsystem (Jakob 2008, S. 28). Um die Registereintragung vollziehen zu können, muss eine Stiftung über eine Mindestkapitalausstattung von 50.000 CHF verfügen. Darüber hinaus haben die Statuten Bestimmungen zur ausschließlichen Verwendung des Vermögens für den Stiftungszweck und ein Verbot des Vermögensrückflusses an die Gründer zu enthalten. Als weitere Voraussetzung zur Registereintragung ist bereits zum Entstehungszeitpunkt eine Revisionsstelle zu benennen (Ferrari-Visca 2008, S. 30).

Das durch die Stiftungsfreiheit geprägte schweizerische Stiftungsrecht legt keine Verwaltungsstrukturen fest, so dass gem. Art. 83 ZGB sämtliche Stiftungsorgane sowie die übrige Stiftungsgestaltung durch die Satzung und somit durch den Stifter alleine bestimmt werden (Jakob 2008, S. 28). Lediglich Art. 83 ZGB bestimmt den Mindestinhalt der Stiftungsurkunde, nach dem die Organe sowie die Verwaltungsart in der Urkunde festzuschreiben sind. Das oberste Stiftungsorgan, gemeinhin als Stiftungsrat oder -vorstand bezeichnet, besorgt das Stiftungsgeschäft, vertritt die Stiftung nach außen und sichert insbesondere die ordnungsgemäße Einhaltung der Satzungsbestimmungen sowie die Verwirklichung des Stifteranliegens. Daneben werden entsprechend der international üblichen Praxis weitere Gremien bestimmt, die für fachspezifische Aufgaben genutzt werden (Lichtsteiner/Degen/Bärlocher 2008, S. 5).

\section{b) Vereinsrecht}

Das schweizerische Vereinsrecht ist analog zum Stiftungsrecht in einem eigenen Abschnitt im ZGB geregelt und verfolgt eine freie Körperschaftsbildung. Demnach können Vereine ohne staatliche Mitwirkung oder Eintragungspflicht gegründet werden und die Rechtsfähigkeit erwerben 
(Hippel 2007, S. 523). Der Verein entsteht gem. Art. 60 Abs. 1 ZGB sobald der Wille, als juristische Person aufzutreten, aus der Satzung erkennbar ist. Der Vereinszweck kann entweder auf gemeinnützige Aufgaben, im Sinne eines Idealvereins gem. Art. 60 Abs. 1 ZGB, oder auf die Erlangung von wirtschaftlichen Vorteilen gem. Art. 59 Abs. 2 ZGB gerichtet sein. Im zuletzt genannten Fall, dem Wirtschaftsverein, untersteht dieser den gesetzlichen Regelungen des schweizerischen Gesellschafts- und Genossenschaftsrechts (Hippel 2007, S. 519-520). Allerdings kann auch ein Idealverein wirtschaftlich, im Rahmen eines kaufmännischen Betriebes tätig sein, wenn dies der Zweckverfolgung dient und keine materiellen Vorteile für die Mitglieder generiert werden. In solch einem Fall werden gem. Art. 61 Abs. 2 Nr. 1 ZGB i. V. m. Art. 957 OR die Vorschriften des OR bezüglich Registereintragung und Buchführung anwendungspflichtig (Hippel 2007, S. 522-523).

Entsprechend zum Stiftungsrecht verzichtet der Gesetzgeber auf eine detaillierte Regelung der Statuteninhalte und normiert in Art. 60 Abs. 2 ZGB lediglich deren Schriftform sowie eine Mindestauskunftspflicht über den Zweck, die Vereinsverwaltung und die finanziellen Mittel. Enthalten die Satzungen keine Anweisung über die Organisationsausgestaltung, wird der Verein nach den Vorschriften des Art. 64 ZGB von der Mitgliederversammlung verwaltet. Diese stellt das beschlussfähige Organ gem. Art. 66-68 ZGB dar und entscheidet über sämtliche Angelegenheiten, die nicht im Rahmen der Statuten auf andere Organe übertragen wurden. Darüber hinaus entscheidet sie gem. Art. 65 Abs. 1, Abs. 2 ZGB über die Aufnahme und den Ausschluss von Mitgliedern, wählt den Vorstand und übt die Kontrolle über sämtliche Organe aus, die sie jederzeit abberufen kann.

\section{Externe Corporate Governance-Instrumente von Stiftungen und Vereinen}

\section{a) Rechnungslegung}

Der Stiftungsrat ist gem. Art. 83 a Abs. 1 ZGB zur kaufmännischen Buchführung unter Anwendung der handelsrechtlichen Buchführungsnormen (Art. 957-963 OR) verpflichtet. Alle im Handelsregister eingetragenen Stiftungen müssen gem. Art. 957 OR über eine ordnungsgemäße, den gesellschaftlichen Erfordernissen angepasste, Buchführung verfügen und gem. Art. 958 Abs. 1 OR jährlich ein Inventar, eine Bilanz sowie eine Betriebsrechnung erstellen. Hierbei handelt es sich um eine Erfolgsrechnung im Sinne einer GuV (Zöbli 2007, S. 90-110). Wird zur Zweckerfüllung ein Gewerbe betrieben, sind gem. Art. 83 a Abs. 2 ZGB i. V. m. Art. 662-663 b und $697 \mathrm{~h}$ OR zusätzlich die für die Aktiengesellschaften geltenden Rechnungslegungs- und Publizitätsvorschriften zu beachten. Hiernach ist jährlich ein Geschäftsbericht gem. Art. 662 OR aufzustellen, der neben einer Jahresrechnung, welche Bilanz, GuV und Anhang umfasst, auch aus einem Jahresbericht besteht. Letzterer stellt gem. Art. 663 d OR den Geschäftsverlauf sowie die wirtschaftliche und finanzielle Lage der Gesellschaft dar und entspricht dem deutschen Lagebericht nach $\S 289$ HGB.

Neben diesen bundesgesetzlichen Vorschriften gibt es zusätzlich einen Rechnungslegungsstandard, den Swiss Generally Accepted Accounting Principles Fachempfehlung zur Rechnungslegung 21 (Swiss GAAP FER), dessen Anwendung jedoch nicht gesetzlich vorgeschrieben ist (Zö- 
bli/Neubert 2009, S. 33-34). Die Swiss GAAP FER stellen Rechnungslegungsempfehlungen dar und werden von der schweizerischen Forschung und Wissenschaft sowie der Praxis veröffentlicht. Sie sind allgemein anerkannt und als Mindeststandard für die Rechnungslegung börsennotierten Unternehmen in der Schweiz anzuwenden (Meyer 2009). Im Rahmen der Fachempfehlung 21 wird eine adäquate Jahresabschlusserstellung speziell für gemeinnützige Unternehmen rechtsformübergreifend geregelt. Zusätzlich zur Anwendung der übrigen Swiss GAAP FER normiert dieser Standard die Erstellung eines Leistungsberichts und einer Kapitalveränderungsrechnung, um den spezifischen organisatorischen Besonderheiten von NPO Rechnung zu tragen (Müller 2009, S. 202-203).

Das schweizerische Recht gibt Vereinen lediglich minimale verbindliche Vorgaben zur Rechnungslegung, Prüfung und Publizität vor. Demnach sind Vereine nur dann zur Führung eines kaufmännischen Rechnungswesens und Jahresabschlusserstellung im Sinne der o.g. Vorschriften des OR über die kaufmännische Buchführung verpflichtet, wenn ein Gewerbe betrieben wird und der Verein ins Handelsregister eingetragen werden muss bzw. eine Eintragung auf freiwilliger Basis stattgefunden hat. Die Eintragung erfolgt gem. Art. 69 a i. V. m. 61 ZGB obligatorisch, wenn der Verein aufgrund seiner Größe revisionspflichtig ist (Donschen 2008, S. 100-101). Ist dies nicht der Fall, hat der Vorstand über das Vermögen sowie die Einnahmen und Ausgaben ordentlich Buch zu führen. Dabei ist die Rechnungslegung an den Umfang und die Komplexität des Geschäfts anzupassen. Ferner sind die Grundsätze ordnungsgemäßer Buchführung (z. B. Vollständigkeit, Klarheit und Übersichtlichkeit) sowohl bei der Aufstellung einer Einnahmen-Ausgaben-Rechnung als auch bei Anwendung der doppelten Buchführung und Bilanzerstellung zu beachten (Madörin/Bertschinger 2009, S. 270-271).

\section{b) Prüfung}

Der Stiftungsrat wählt gem. Art. 83 b Abs. 1 ZGB zur periodischen und externen Rechnungslegungsprüfung eine unabhängige, staatlich beaufsichtigte Revisionsstelle, die dem Rat über ihre Prüfungstätigkeit schriftlich Bericht zu erstatten hat (BMJ 2001, Anlage 3, S. 12). Diese sog. ordentliche Revision nach den aktienrechtlichen Vorschriften ist gem. Art. 83 b Abs. 3 i. V. m. Art.727-731 OR für jede Stiftung verpflichtend durchzuführen, wenn gem. Art. 727 Abs. 1 Nr. 2 a-c OR die Bilanzsumme mindestens 20 Mio. CHF, die Umsatzerlöse mindestens 40 Mio. CHF betragen und/oder die Stiftung durchschnittlich über wenigstens $250 \mathrm{Ar}-$ beitnehmer verfügt. Zur Auslösung der Prüfungspflicht genügt gem. Art. 727 Abs. 1 Nr. 2 OR eine Überschreitung von jeweils zwei der drei Schwellenwerte in zwei aufeinanderfolgenden Geschäftsjahren. Stiftungen können auf Antrag des Stiftungsvorstandes befreit werden, wenn die Bilanzsumme 200.000 CHF nicht überschreitet und die Stiftung nicht zu öffentlichen Spenden aufruft (Art. 1 Abs. 1 Verordnung über die Revisionsstelle von Stiftungen).

Erfüllt eine Stiftung die vorgenannten Schwellenwerte nicht und wurde sie trotz geringer Größe nicht von der Revisionspflicht befreit, wird gem. Art. 83 Abs. 2 OR eine eingeschränkte Revision im Sinne von Art. 727 a OR i. V. m. Art. 729-729 c OR durchgeführt. Im Gegensatz zu einer vollständigen Revision wird hierbei gem. Art. 729 a Abs. 1 Nr. 1 OR lediglich kontrolliert, ob Sachverhalte vorliegen, die erkennen lassen, dass die Rechnungslegung nicht den satzungs-/ge- 
setzmäßigen Ansprüchen entspricht (Müller 2009, S. 205). Die Durchführung einer ordentlichen Prüfung im Sinne des OR von zur eingeschränkten Revision verpflichteten Stiftungen kann jedoch gem. Art. 83 b Abs. 4 ZGB von der Stiftungsaufsicht verlangt werden, wenn sie dies zur Einschätzung der wirtschaftlichen Lage für geboten hält. Daneben können die Stiftungsstatuten gem. Art. 727 Abs. 3 OR eine freiwillige Prüfungsanordnung zur ordentlichen bzw. bei vollständiger Befreiung auch zur eingeschränkten Revision enthalten. Nach der Prüfung des Revisionsberichtes durch den Stiftungsrat ist das Rechnungswesen periodisch von diesem zu genehmigen (Zöbli/ Neubert 2009, S. 21). Abbildung 8 zeigt die Revisionspflichten von Stiftungen inkl. ihrer Wahlmöglichkeiten (sog. Opting-System) auf (Wagner/Plüss 2008, S. 206-207).

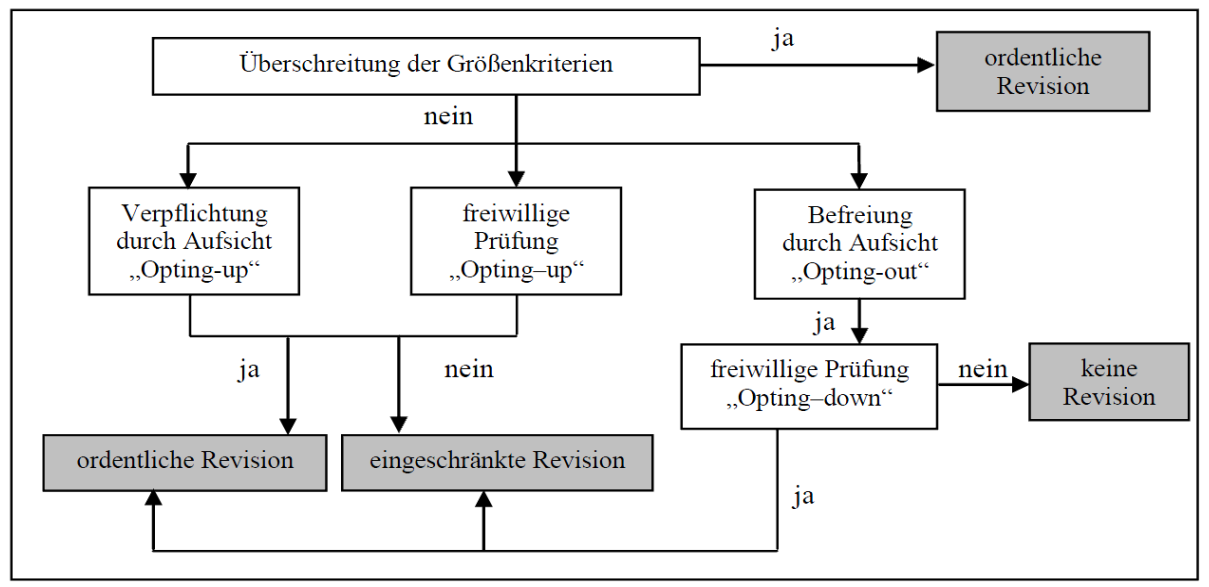

Abbildung 4: Revisionspflichten schweizerischer Stiftungen

Quelle: Eigene Darstellung

Die Prüfungspflicht von Vereinen wird explizit in Art. 69 b ZGB geregelt. Demnach unterliegt ein Verein gem. Art 69 b Abs. 1, Abs. 3 ZGB bei Überschreitung bestimmter Größenkriterien (Bilanzsumme $\geq 10$ Mio. CHF, Umsatz $\geq 20$ Mio. CHF und Vollzeitstellen $\geq 50$ ) der ordentlichen Prüfungspflicht. Diese Revisionspflicht löst gleichfalls eine Eintragungspflicht im Handelsregister gem. Art. 61 Abs. 1 Nr. 2 ZGB aus. Erreicht ein Verein die vorausgesetzten Größenkriterien nicht, muss er sich dennoch gem. Art. 69 b Abs. 2 ZGB einer eingeschränkten Prüfung unterziehen, wenn dies von einem persönlich haftenden Mitglied verlangt wird. Zusätzlich sind die aktienrechtlichen Revisionsvorschriften des OR aufgrund des Verweises in Art. 69 b Abs. 3 ZGB auch auf Vereine anwendbar. Darüber hinaus können die Statuten oder auch die Mitgliederversammlung gem. Art. 69 b Abs. 4 ZGB und Art. 727 Abs. 3 OR eine Rechnungslegungsprüfung anordnen. 


\section{c) Publizität}

Aufgrund des liberal ausgestalteten Stiftungsrechtes und der damit einhergehenden Freizügigkeit unterliegen die schweizerischen Stiftungen geringen Publizitätspflichten. So besteht lediglich für unter der Aufsicht des Bundes stehende Stiftungen die Pflicht, sich in ein öffentlich zugängliches Stiftungsverzeichnis eintragen zu lassen (Lichtsteiner/Degen/Bärlocher 2008, S. 13). Andere Stiftungen haben, außerhalb der obligatorischen Eintragung in das Handelsregister, keine Publizitätspflichten. Zu den in das Handelsregister eintragungspflichtigen und gem. Art. 930 OR öffentlich zugänglichen Informationen zählen nach Art. 927-943 OR der Name, Zweck und Sitz sowie die Besetzung der Stiftungsorgane. Die Folge ist, dass kaum Transparenz hinsichtlich schweizerischer Stiftungen und ihrer wirtschaftlichen Lage herrscht. Lediglich die Stiftungsaufsichtsbehörden haben im Rahmen ihrer Kontrollaufgabe die Pflicht zur Einsichtnahme in die Bücher und die finanzielle Situation der Stiftungen (Lichtsteiner/Degen/Bärlocher 2008, S. 3). Die einzige Ausnahme stellen Stiftungen dar, die einen wirtschaftlichen Geschäftsbetrieb führen. Aufgrund der gem. Art. 83 a Abs. 2 ZGB i. V. m. Art. 697 h Abs. 2 OR verpflichtenden Anwendung weitergehender aktienrechtlichen Vorschriften müssen diese Stiftungen im Falle von berechtigtem Interesse schutzwürdiger Personen Einsicht in ihre Jahresrechnung und den Revisionsbericht gewähren. Allerdings normiert Swiss GAAP FER 21 als Empfehlung die Offenlegung eines Kommentars zur Jahresrechnung und die Publizität des Leistungsberichtes, der in verbaler Form über die Zweckerreichung, Wirtschaftlichkeit des Ressourceneinsatzes, Leistungsfähigkeit der Organisation sowie das Stiftungsumfeld berichten soll (Zöbli 2007, S. 38, 131-133).

Die gem. Art. 61 Abs. 2 ZGB eintragungspflichtigen Vereine haben ebenfalls die Rechtsvorschriften zu beachten, die für eingetragene Stiftungen gelten, da hier die allgemeinen Vorschriften zum Handelsregister des OR Anwendung finden. Darüber hinaus bestehen für schweizerische Vereine keine Pflichten zur Offenlegung des Jahresabschlusses oder sonstiger relevanter Daten.

\section{d) Aufsicht}

Die Aufsicht über Stiftungen übernimmt das Gemeinwesen in der Schweiz (Pavel 1971, S. 68). Somit unterstehen Stiftungen gem. Art. 84 Abs. 1 ZGB entsprechend ihres Aufgabengebietes dem Bund, den Kantonen oder den Kommunen, in denen sie ihren Sitz haben. Die staatliche Rechtsaufsicht umfasst nach Art. 84 Abs. 2 ZGB die Überwachung der ordnungsgemäßen Zweckverwirklichung und Mittelverwendung sowie die Einhaltung der Statuten durch den Stiftungsrat und schreitet bei Missbrauch und Gesetzübertretungen ein (Zöbli/Neubert 2009, S. 20). Neben diesen Tätigkeiten sind die Stiftungsaufsichtsbehörden für die Genehmigung von Satzungsänderungen und Stiftungsauflösungen zuständig und stehen den Stiftern zusätzlich als Beratungsinstanz zur Verfügung (Ferrari-Visca 2008, S. 30). Die Mittel der Aufsichtsbehörden zur Wahrnehmung ihrer Aufgaben reichen von Ermahnungen über Anweisungen bis hin zur Stiftungsauflösung. Ist eine Mängelbehebung hinsichtlich der Gewährleistung einer ordentlichen und funktionsfähigen Verwaltung aussichtslos, ist die Behörde gem. Art. 83 d Abs. 2 ZGB ermächtigt, das Stiftungsvermögen einer anderen, zweckähnlichen Stiftung zukommen zu lassen. Darüber hinaus sind die Regionalbehörden befugt, die Stiftungsorganisation gem. Art. 85 ZGB zu ändern, um eine zweck- 
mäßige Tätigkeit und den Erhalt des Stiftungsvermögens zu gewährleisten sowie gem. Art. 86 ZGB das Stiftungsziel aufgrund entwicklungsbedingter Differenzen zwischen originärem Zweck und Stifterwillen zu korrigieren (Lichtsteiner/Degen/Bärlocher 2008, S. 6).

Darüber hinaus haben Stiftungen gegenüber den Aufsichtsbehörden eine umfangreiche Berichterstattungspflicht. Im Rahmen dieser vorbeugenden Kontrolltätigkeit verlangt die schweizerische Stiftungsaufsicht jährlich die Vorlage der Jahresrechnung, den Prüfbericht der Revision inkl. der Genehmigung des Jahresabschlusses durch den Stiftungsrat sowie einen umfassenden Tätigkeitsbericht (Ferrari-Visca 2008, S. 31; BMJ 2001, Anlage 3, S. 12). Revisionsbefreite Stiftungen unterliegen einer strengeren Aufsicht durch die Stiftungsaufsicht. In diesem Rahmen können zusätzliche Unterlagen, wie bspw. Kreditoren- und Debitorenlisten oder Saldenbestätigung von Kreditinstituten, eingefordert werden (Zöbli/Neubert 2009, S. 25). Zudem ist der Stiftungsprüfer nach Art. 83 c ZGB verpflichtet, den Aufsichtsbehörden im Rahmen der Abschlussprüfung eine Abschrift des Revisionsberichtes sowie sämtliche wichtige Mitteilungen an die Stiftungen zu übermitteln. Stellt das oberste Stiftungsorgan oder die Revisionsstelle eine wirtschaftliche Schieflage der Organisation in Form von Überschuldung oder Zahlungsunfähigkeit fest, ist der Aufsichtsbehörde eine Zwischenbilanz vorzulegen. Entsprechend ihrer Befugnisse kann sie Gegenmaßnahmen anordnen oder im Falle einer Untätigkeit der Organisationsvorstände durchführen lassen. Die Gewalt der Stiftungsbehörde reicht gem. Art. 84 a ZGB bis hin zur Eröffnung eines Konkursverfahrenes gegen die Stiftung. Diese im Rahmen der Reform eingeführten Regelungen dienen dem Gläubigerschutz, da schweizerische Stiftungen grundsätzlich keinen Publizitätspflichten unterliegen und sich die Gläubiger über die finanzielle Stiftungslage nicht eigenständig informieren können (Lichtsteiner/Degen/Bärlocher 2008, S. 7). Ferner stellt diese staatliche Überwachung sicher, dass die Stiftungsmittel zweckgemäße Verwendung finden (Pavel 1971, S. 69). Wie auch in Deutschland unterstehen schweizerische Vereine, mit Ausnahme einer staatlichen Rechtsaufsicht keiner weiteren rechtsformspezifischen Aufsicht.

\section{Fazit}

Im Vergleich zu den Größenkriterien der deutschen Rechnungslegungspflicht gem. des PublG zeigt sich, dass der österreichische und schweizerische Gesetzgeber auf eine adäquate Berichterstattung und erhöhte Kontrollen im Vereins- und Stiftungssektor hinwirkt, erkennbar an den unabdingbaren Pflichten zur kaufmännischen Rechnungslegung und Revision der erstellten Jahresabschlüsse.

In Österreich werden die Stiftungen explizit den Kaufleuten gleichgestellt, um eine Umgehung der kaufmännischen Vorschriften durch Gründung eigennütziger Stiftungen zu vermeiden. Im Vereinsrecht nimmt der österreichische Gesetzgeber auf die finanziellen Verhältnisse Rücksicht und bindet die Rechnungslegungs- und Prüfungspflichten an die Erfüllung von Größenkriterien. Sowohl in Deutschland, Österreich und der Schweiz legen die Gesetze nur ein geringes Augenmerk auf die Publizität. So bestehen lediglich geringe Pflichten zur Eintragung in öffentliche Register, es wird keine allgemeine Offenlegung der Rechnungslegung gefordert und die Veröffentlichungspflicht wird grundsätzlich an dem wirtschaftlichen Tätigkeitsumfang der Organisationen ausgerichtet. Personen mit berechtigtem Interesse können zwar zum Großteil Einblick in 
die finanzielle Situation verlangen, allerdings reicht dies nicht aus, um auch das Auskunftsinteresse der öffentlichen Allgemeinheit zu befriedigen. Abbildung 5 stellt die gesetzlich verankerten Unterschiede hinsichtlich der Rechnungslegungs-, Prüfungs- und Publizitätspflicht zwischen Deutschland, Österreich und der Schweiz zusammenfassend dar.

\begin{tabular}{|c|c|c|c|c|c|c|c|c|}
\hline & & \multicolumn{2}{|c|}{ Rechnungslegungspflicht } & \multicolumn{4}{|c|}{ Prüfungspflicht } & \multirow{2}{*}{$\begin{array}{c}\text { externe } \\
\text { Publizitätspflicht der } \\
\text { Rechnungslegung }\end{array}$} \\
\hline & & $\begin{array}{l}\text { EAR inkl. } \\
\text { Vermögensüibersicht }\end{array}$ & $\begin{array}{c}\text { kaufmännisch eingerichtetes } \\
\text { Rechnungswesen }\end{array}$ & $\begin{array}{l}\text { Interne Rechnungs- } \\
\text { /Kassenprüfer }\end{array}$ & \multicolumn{3}{|c|}{$\begin{array}{l}\text { Wirtschaftsprüfer/ } \\
\text { Revisionsstellen }\end{array}$} & \\
\hline \multirow{3}{*}{ 总 } & Deutschland & Pflicht & $\begin{array}{l}\text { Gewerbebetrieb von Stiftungen } \\
\text { mit HR-Eintragung }\end{array}$ & - & \multicolumn{3}{|c|}{$\begin{array}{c}\text { Stiftungen mit Gewerbebetrieb } \\
\text { BS }>65 \text { Mio. } €, \\
\text { Umsatz }>130 \text { Mio. } €, \\
\text { Arbeitnehmer }>5.000\end{array}$} & $\begin{array}{c}\text { Stiftungen mit } \\
\text { Gewerbebetrieb } \\
\text { BS }>65 \text { Mio. } €, \\
\text { Umsatz }>130 \text { Mio. } €, \\
\text { Arbeitnehmer }>5.000\end{array}$ \\
\hline & Österreich & $\cdot$ & Pflicht & - & \multicolumn{3}{|c|}{ Pflicht } & $\begin{array}{c}\text { Pflicht auf Anfrage der } \\
\text { Destinatäre }\end{array}$ \\
\hline & Schweiz & $\cdot$ & Pflicht & $\cdot$ & $\begin{array}{c}\text { Pflicht } \\
\text { BS }>20 \text { Mio. } € \\
\text { Umsatz }>40 \text { Mio. } € \\
\text { Vollzeitstellen }>250\end{array}$ & \begin{tabular}{|c|} 
sonst: \\
eingeschränkte \\
Revision
\end{tabular} & \begin{tabular}{|c|} 
Befreiung \\
BS $<131.260 €$ \\
keine \\
öffentlichen \\
Spendenaufrufe \\
\end{tabular} & $\begin{array}{c}\text { Stiftungen mit } \\
\text { Gewerbebetrieb } \\
\text { Pflicht auf Anfrage und } \\
\text { schutzwürdigem } \\
\text { Interesse }\end{array}$ \\
\hline \multirow{3}{*}{ 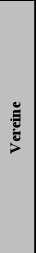 } & Deutschland & Pflicht & $\begin{array}{c}\text { Gewerbebetrieb von Vereinen mit } \\
\text { HR-Eintragung }\end{array}$ & - & \multicolumn{3}{|c|}{$\begin{array}{c}\text { Wirtschaftsvereine } \\
\text { BS } \geq 65 \text { Mio. } €, \\
\text { Umsatz } \geq 130 \text { Mio. } €, \\
\text { Arbeitnehmer } \geq 5.000\end{array}$} & $\begin{array}{c}\text { Wirtschaftsvereine } \\
\text { BS }>65 \text { Mio. } €, \\
\text { Umsatz }>130 \text { Mio. } €, \\
\text { Arbeitnehmer }>5.000\end{array}$ \\
\hline & Österreich & $\begin{array}{l}\text { kleine Vereine } \\
\text { Eimnahmen/Ausgaben } \\
<1 \text { Mio. } €\end{array}$ & $\begin{array}{c}\text { mittelgroße \& große Vereine } \\
\text { Einnahmen/Ausgaben } \\
>1 \text { bzw. } 3 \text { Mio. } \epsilon \\
\text { Spenden }>1 \text { Mio. } €\end{array}$ & $\begin{array}{c}\text { kleine \& mittelgroBe } \\
\text { Vereine } \\
\text { Einnahmen/Ausgaben } \\
<3 \text { Mio. } €\end{array}$ & \multicolumn{3}{|c|}{$\begin{array}{c}\text { große Vereine } \\
\text { Einnahmen/Ausgaben }>3 \text { Mio. } € \\
\text { Spendeneinnahmen }>1 \text { Mio. } €\end{array}$} & - \\
\hline & Schweiz & Pflicht & $\begin{array}{c}\text { Vereine mit Gewerbebetrieb und } \\
\text { HR-Eintragung }\end{array}$ & - & $\begin{array}{c}\text { Pflicht } \\
\text { BS } \geq 6,6 \text { Mio. } € \\
\text { Umsatz } \geq 13,1 \text { Mio. } € \\
\text { Vollzeitstellen } \geq 50\end{array}$ & \multicolumn{2}{|c|}{$\begin{array}{l}\text { eingeschränkte Revision auf } \\
\text { Verlangen eines persönlich } \\
\text { haftenden Mitgliedes }\end{array}$} & $\begin{array}{c}\text { Vereine mit } \\
\text { Gewerbebetrieb } \\
\text { Pflicht auf Anfrage unc } \\
\text { schutzwürdigem }\end{array}$ \\
\hline
\end{tabular}

Abbildung 5: Überblick über die externen Corporate Governance-Vorschriften von Deutschland, Österreich und der Schweiz

Quelle: Eigene Darstellung

Insgesamt zeigt sich, dass Deutschland im internationalen Vergleich geringe Standards zur Ausgestaltung der externen Überwachung von NPO aufweist. Darüber hinaus sind die Verpflichtungen lediglich auf den Gewerbebetrieb bezogen und unterliegen zum Teil derart hohen Schwellenwerten, dass diese Vorschriften in der Praxis kaum Anwendung finden. Die gesetzlichen Verpflichtungen zur Ausgestaltung der Finanzverfassung und Offenlegung sowie Prüfung sind vor dem Hintergrund des Wachstums dieses Sektors und der diesbezüglich unzureichenden Informationsabgabe über die wirtschaftliche Lage der Vereine und Stiftungen nicht mehr ausreichend. Zur notwendigen Stärkung des Vertrauens in gemeinnützige Organisationen und aufgrund der auch in diesem Sektor fortschreitenden Internationalisierung (Hopt 2006, S. 162; Wilke 2004, S. 90) sind daher verstärkte Anforderungen an das externe Rechnungswesen, die Transparenz und die Revision der Daten unerlässlich, um die Organisationen des Dritten Sektors konkurrenzfähig zu halten.

Dies haben bereits die Gesetzgeber anderer Länder erkannt und setzen in ihren Reformen zum Gemeinnützigkeitsrecht verstärkt auf angemessene und verlässliche Berichterstattung. Der internationale Vergleich hat herausgestellt, dass in Deutschland umfassende Reformen notwendig sind. Die internationalen Regelungen, vor allem die des österreichischen Vereinsrechtes könnten dabei als Leitlinien dienen, da hier ein annehmbarer Kompromiss zwischen dem Kontrollbedarf und dem Schutz der NPO-Interessen gefunden worden ist. Die bestehende staatliche Rechtsaufsicht über Vereine und Stiftungen wird in Deutschland als ausreichend erachtet, da insbesondere 
der Gemeinnützigkeitsstatus und die damit einhergehenden Steuervorteile durch das Finanzamt kontrolliert werden. Allerdings sollte die Stiftungsaufsicht vereinheitlicht werden, um Vereinfachungen und Rechtssicherheit zu schaffen.

Abstract

Ira Führer and Remmer Sassen; External corporate governance elements of nonprofit foundations and associations in an international context. Implications from Austrian and Swiss law for reform approaches in Germany

nonprofit governance, nonprofit association, nonprofit foundation, financial reporting, auditing, publicity, public monitoring

This paper shows reform approaches for external corporate governance elements of nonprofit foundations and associations. German Law prescribes only low requirements on financial accounting, auditing, publicity and public monitoring. Therefore the paper analyses the corresponding requirements in Austria and Switzerland to receive implications for reform approaches in Germany. As a result it is shown that an appropriate and reliable reporting is necessary to enforce confidence in nonprofit organizations.

\section{Literaturverzeichnis}

Anheier, H. K./Priller, E./Seibel, W./Zimmer, A. (2007), Der Nonprofit Sektor in Deutschland, in: Badelt, C./Meyer, M./Simsa, R. (Hrsg.), Handbuch der Nonprofit Organisation. Strukturen und Management, 4. Aufl., Stuttgart, S. 17-39.

BMJ (2001), Bundesministerium der Justiz, Bericht der Bund-Länder-Arbeitsgruppe Stiftungsrecht vom 19.10.2001, abrufbar unter: http://www.bmj.bund.de/files/-/405/Abschlussbericht.pdf (18.6.2010), S. 1-177.

Brändle, C./Schnetzer, M. (2002), Das österreichische Vereinsrecht. Rechtliche Grundlagen - Steuerliche Aspekte - Vereinsgesetz 2002 - Vereinsrichtlinien 2001, 3. Aufl., Wien.

Donschen, A. (2008), Die genossenschaftliche Pflichtprüfung. Vergleich mit Vereins- und Aktienrecht, Aachen.

Doralt, P. (1996), Die Österreichische Privatstiftung. Ein neues Gestaltungsinstrument für Unternehmen, in: ZGR, 25. Jg., S. 1-17.

Ebers, M. (2005), Die genossenschaftliche und vereinsrechtliche Prüfung in Österreich und in der Schweiz - Reformvorschläge für das deutsche Genossenschafts- und Vereinsrecht, in: Walz, W. R./ Bösche, B. (Hrsg.), Wie viel Prüfung braucht der Verein - wie viel Prüfung verträgt die Genossenschaft?, Hamburg, S. 55-84.

Ferrari-Visca, B. (2008), Stiftungsaufsicht. Rolle und Aufgaben in der Schweiz, in: S\&S, 11. Jg, S. 30-31.

Freidank/Velte, (2008), Einfluss der Corporate Governance auf die Weiterentwicklung von Controlling und Interner Revision, in: Freidank/Peemöller (Hrsg.), Corporate Governance und Interne Revision. Handbuch für die Neuausrichtung des Internal Auditings, S. 711-745.

Führer/Sassen (2010), Anforderungen an externe Corporate-Governance-Strukturen in Stiftungen. Reformbedarf des Stiftungsrechts hinsichtlich Rechnungslegung, Prüfung und Aufsicht, in: ZCG, 5. Jg., S. 227-236.

Grünberger, H. (2004), Reformierte Vereinsrechnungslegung in Österreich, in: Walz, W. R. (Hrsg.), Rechnungslegung und Transparenz im Dritten Sektor, München, S. 119-127.

Hippel, T. v. (2007), Grundprobleme von Nonprofit-Organisationen. Eine zivilrechts-dogmatische, steuerrechtliche und rechtsvergleichende Untersuchung über Strukturen, Pflichten und Kontrollen und wirtschaftliche Tätigkeit von Vereinen und Stiftungen, Tübingen.

Hopt, K. J. (2005), Corporate Governance in Nonprofit-Organisationen, in: Hopt, K. J./Hippel, T. v./Walz, W. R. (Hrsg.), Nonprofit-Organisationen in Recht, Wirtschaft und Gesellschaft. Theorien - Analysen - Corporate Governance, Tübingen, S. 243-258.

Hopt, K. J. (2006), Die Europäische Stiftung - Ein Plädoyer für eine neue europäische Rechtsform!, in: EuZW, 17. Jg., S. 162. 
Jakob, D. (2008), Status quo und Perspektiven. Das Schweizer Stiftungsrecht, in: S\&S, 11. Jg., S. 28-29.

Lang, M./Rief, R. (1994), Die österreichisches Privatstiftung, in: Die Bank, o.Jg., S. 147-152.

Lichtsteiner, H./Degen, C./Bärlocher, C. (2008), Stiftungslandschaft Schweiz. Tatsachen und Recht, in: Rote Seiten, Beilage zu S\&S, 11. Jg., H. 4, S. 2-23.

Löwe, M. (2003), Rechnungslegung von Nonprofit-Organisationen. Anforderungen und Ausgestaltungsmöglichkeiten unter Berücksichtigung der Regelungen in Deutschland, USA und Großbritannien, Berlin.

Madörin, B./Bertschinger, P. (2009), Rechnungslegung und Wirtschaftsprüfung/Accounting and Auditing in Switzerland, Bern 2009.

Meyer, C. (2009), Swiss GAAP FER. Erläuterungen, Illustrationen und Beispiele, Zürich.

Müller, K. (2009), Rechnungslegung für Nonprofit-Organisationen. Swiss GAAP FER 21, in: ST, 83. Jg., S. 202-206.

Nowotny, C./Fida, S. (2007), Rechtliche Gestaltungsformen für NPOs, in: Badelt, C./Meyer, M./Simsa, R. (Hrsg.), Handbuch der Nonprofit Organisation. Strukturen und Management, 4. Aufl., Stuttgart, S. 202-230.

o.V. (2007/2008), Österreich. Stiftungsreich, in: Die Stiftung 2./3. Jg., S. 53-54.

Paetzmann, K. (2007), Corporate Governance, in Freidank, C.-Chr./Lachnit, L./Tesch, J. (Hrsg.), Vahlens Großes Auditing Lexikon, München, S. 302-304.

Pavel, U. (1971), Schweiz, in: Stifterverband für die Deutsche Wissenschaft (Hrsg.), Schriften zum Stiftungswesen. Stiftungen in Europa. Eine vergleichende Übersicht, Band 5, Essen-Bredeney, S. 61-98.

Priller, E./Zimmer, A. (2001), Der Dritte Sektor: Wachstum und Wandel. Aktuelle Deutsche Trends, Gütersloh.

Purtschert, R./Schnurbein, G. v./Beccarelli, C. (2006), Gemeinnützige Stiftungen in der Schweiz - Zwischen Aufbruch und Bewahrung, in: Egger, P./Helmig, B./Purtschert, R. (Hrsg.), Stiftung und Gesellschaft. Eine komparative Analyse des Stiftungsstandortes Schweiz. Deutschland, Liechtenstein, Österreich, USA, Basel, S. 91-118.

Schneider, J./Bienek, H. J. (2004), Corporate Governace - Etymologie und Theorieentwicklung, in: Voggensperger, R. C./Bienek, H. J./Schneider, J./Thaler, G. O. (Hrsg.), Gutes besser tun. Corporate Governance in NonprofitOrganisationen, Bern/Stuttgart/Wien, S. 25-44.

Schneider, J./Minnig, C./Freiburghaus, M. (2007), Strategische Führung von Nonprofit-Organisationen, Bern.

Simsa, R. (2006), Stiftungen in Österreich - Vernachlässigt und umstritten, in: Egger, P./Helmig, B./Purtschert, R. (Hrsg.), Stiftung und Gesellschaft. Eine komparative Analyse des Stiftungsstandortes Schweiz. Deutschland, Liechtenstein, Österreich, USA, Basel, S. 139-155.

Strachwitz, R. (2004), Verschwiegenheit und Transparenz gemeinwohlorientierter Aktuere, in: Walz, W. R. (Hrsg.), Rechnungslegung und Transparenz im Dritten Sektor, München, S. 203-214.

Wagner, J./Plüss, A. (2008), Entwicklungen im schweizerischen Wirtschafts- und Steuerrecht, in: RIW, 54. Jg., S. 205-211.

Walz, W. R. (2002), Die Selbstlosigkeit gemeinnütziger Non-Profit-Organisationen im Dritten Sektor zwischen Staat und Markt, in: JZ, 57. Jg., S. 268-275.

Walz, W. R. (2005), Rechnungslegung für Nonprofit-Organisationen, in: Hopt, K. J./Hippel, T. v./Walz, W. R. (Hrsg.), Nonprofit-Organisationen in Recht, Wirtschaft und Gesellschaft. Theorien - Analysen - Corporate Governance, Tübingen, S. 259-281.

Werder, A. v. (2003), Ökonomische Grundfragen der Corporate Governance, in: Hommelhoff, P./Hopt, K. J./Werder, A. v. (Hrsg.), Handbuch Corporate Governance. Leitung und Überwachung börsennotierter Unternehmen in der Rechts- und Wirtschaftspraxis, Köln, S. 3-27.

Wilke, B. (2004), DZI Spenden-Siegel - Konzept, Erfahrungen und internationaler Vergleich, in: Walz, W. R. (Hrsg.), Rechnungslegung und Transparenz im Dritten Sektor, München, S. 177-191.

Zöbli, D. (2007), Rechnungslegung für Nonprofit-Organisationen. Ein praktischer Kommentar zu Swiss GAAP FER 21, Zürich.

Zöbli, D./Neubert, L. (2009), Jahresabschluss und Finanzen von Stiftungen. Rechnungslegung, Revision, Internes Kontrollsystem (IKS), Sanierung und Vermögenslage, Zürich.

Verzeichnis der Gesetze, Gesetzesentwürfe und Verordnungen

ArbVG: Arbeitsverfassungsgesetz vom 15.1.1974, BGB1. I für die Republik Österreich 1974, S. 393-434 (Rechtsstand vom 13.11.2007).

BAO: Bundesgesetz vom 28.6.1961, betreffend allgemeine Bestimmungen und das Verfahren für die von den Abgabenbehörden des Bundes verwalteten Abgaben (Bundesabgabenordnung - BAO), BGB1. I für die Republik Österreich 1961, S. 1031-1078 (Rechtsstand vom 18.12.2001).

FBG: Firmenbuchgesetz (FBG) vom 11.1.1991, BGBl. I für die Republik Österreich, S. 53-70.

OR: Bundesgesetz betreffend die Ergänzung des Schweizerischen Zivilgesetzbuches (Fünfter Teil: Obligationenrecht) vom 30.3 .1911 (Rechtsstand vom 1.1.2010), abrufbar unter: http://www.admin.ch/ch/d/sr/2/220.de.pdf (18.6.2010), S. 1-502.

StGG: Staatsgrundgesetz vom 21.12.1867 über die allgemeinen Rechte der Staatsbürger für die im Reichsrate vertretenen Königreiche und Länder - StGG, abrufbar unter: http://www.internet4jurists.at/gesetze/stgg.htm (18.6.2010), S. 1-3.

UGB: Bundesgesetz über besondere zivilrechtliche Vorschriften für Unternehmen (Unternehmensgesetzbuch UGB) vom 13.9.1897, abrufbar unter: http://www.ris.bka.gv.at/GeltendeFassung.wxe?Abfrage=Bundesnormen\&Gesetzesnummer=10001702 (18.6.2010), S. 1-161. 
VereinsRL: Richtlinien für die Besteuerung von Vereinen (VereinsRL), abrufbar unter: https://findok.bmf.gv.at/ findok/showBlob.do;jsessionid=39AB76E53F011B9787B8EB48DCE9D428?base $=$ GesPdf\&rid=13 (18.6.2010), S. 1-236.

VerG: Bundesgesetz über Vereine (Vereinsgesetz 2002 - VerG), abrufbar unter: http://www.sportministerium.at/ files/doc/Stand-bis-02-2009/Gesetze/Bund/Vereinsgesetz.pdf (18.6.2010), S. 1-13.

VO über die Revisionsstelle von Stiftungen: Verordnung über die Revisionsstelle von Stiftungen vom 24.8.2005, abrufbar unter: http://www.admin.ch/ch/d/as/2005/4555.pdf (18.6.2010), S. 1-2.

ZGB: Schweizerisches Zivilgesetzbuch (ZGB) vom 10.12.1907 (Rechtsstand vom 1.2.2010), abrufbar unter: http:// www.admin.ch/ch/d/sr/2/210.de.pdf (18.6.2010), S. 1-350. 